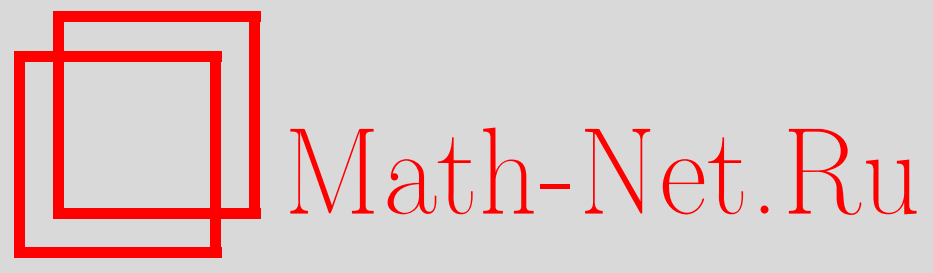

Л. Н. Кривоносов, В. А. Лукьянов, Эрмитовы метрики с (анти)автодуальным тензором Римана, Вестн. Сам. гос. техн. ун-та. Сер. Физ.-мат. науки, 2021, номер 4, 616-633

DOI: https://doi.org/10.14498/vsgtu1867

Использование Общероссийского математического портала MathNet.Ru подразумевает, что вы прочитали и согласны с пользовательским соглашением

http: //www . mathnet.ru/rus/agreement

Параметры загрузки:

IP : 54.89 .56 .158

26 апреля 2023 г., 15:31:03

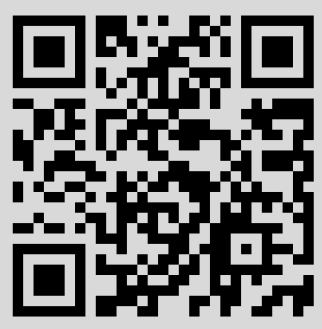


Вестн. Сам. гос. техн. ун-та. Сер. Физ.-мат. науки. 2021. Т. 25, № 4 . С. $616-633$ ISSN: 2310-7081 (online), 1991-8615 (print)

УДК 514.756

\title{
Эрмитовы метрики с (анти)автодуальным тензором Римана
}

\section{Л. Н. Кривоносов, В. А. Лукъянов}

Нижегородский государственный технический университет им. Р. Е. Алексеева, Россия, 603600, Нижний Новгород, ул. Минина, 24.

\begin{abstract}
Аннотация
Составлены уравнения (анти)автодуальности для компонент связности Леви-Чивита (а не для тензора Римана) положительно определенной эрмитовой метрики. Этим известным приемом получается более простая система дифференциальных уравнений в частных производных, влекущая (анти)автодуальность тензора Римана. Эта система 1-го порядка, тогда как уравнения (анти)автодуальности тензора Римана - 2-го порядка. Однако этим способом можно получить лишь часть решений уравнений (анти)автодуальности тензора Римана. Составленные уравнения оказались существенно разными в автодуальном и антиавтодуальном случаях. В случае автодуальности уравнения разбиваются на три класса, для каждого из которых найдено общее решение. В антиавтодуальном случае мы общего решения не нашли, но привели две серии частных решений. Известно, что из (анти)автодуальности тензора Римана вытекает равенство нулю тензора Риччи. Следовательно, найдены пять серий новых решений вакуумных уравнений тяготения Эйнштейна, причем все решения в квадратурах или в явном виде. Указана связь найденных решений с кэлеровыми метриками. В случае (анти)автодуальности связности Леви-Чивита для эрмитовой метрики приведен общий вид параллельных почти комплексных структур, сохраняющих метрику. Они все без кручения. Для произвольной положительно определенной 4-метрики найден общий вид почти комплексных структур, сохраняющих эту метрику.
\end{abstract}

Ключевые слова: (анти)автодуальность, оператор Ходжа, вакуумные уравнения тяготения Эйнштейна, тензор Римана, эрмитова, кэлерова, гиперкэлерова метрика.

Получение: 16 июня 2021 г. / Исправление: 18 сентября 2021 г. Принятие: 12 октября 2021 г. / Публикация онлайн: 16 ноября 2021 г.

\section{Научная статья}

(c) Коллектив авторов, 2021

(c) СамГТУ, 2021 (составление, дизайн, макет)

(웅 Контент публикуется на условиях лицензии Creative Commons Attribution 4.0 International (https://creativecommons.org/licenses/by/4.0/deed.ru)

\section{Образец для цитирования}

Кр и воносов Л. Н., Лукьянов В. А. Эрмитовы метрики с (анти)автодуальным тензором Римана // Вестн. Сам. гос. техн. ун-та. Сер. Физ.-мат. науки, 2021. Т. 25, № 4. C. 616-633. https://doi.org/10.14498/vsgtu1867.

\section{Сведения об авторах}

Леонид Николаевич Кривоносов (1) https://orcid.org/0000-0002-3533-9595 кандидат физико-математических наук; доцент; каф. прикладной математики; e-mail: 1.n.krivonosov@gmail.com

Вячеслав Анатольевич Луквянов (1) https://orcid.org/0000-0002-7294-0232 кандидат физико-математических наук; доцент; каф. прикладной математики; e-mail: oxyzt@ya.ru 
Введение. Отыскание решений вакуумных уравнений Эйнштейна $R_{i j}=0$ не потеряло актуальности до настоящего времени. Помимо многочисленных геометрических применений [1] метрики Эйнштейна находят новые применения и в физике. Например, в теории магнитных монополей фундаментальную роль играет одна из антиавтодуальных метрик Эйнштейна, инвариантная относительно группы $S O(3)$ (см. [2]). Напрямую найти общее решение вакуумных уравнений Эйнштейна не удается, хотя частных решений найдено большое количество (см. [3]). Для отыскания других частных решений существенных успехов удалось добиться обходными путями. Один из них твисторный метод Пенроуза. Другой путь - использование уравнений (анти)автодуальности (проще говоря - дуальности) тензора Римана [4-6]. Метрики, полученные последним способом, называются (анти)автодуальными метриками Эйнштейна. Дифференциальные уравнения дуальности тензора Римана тоже достаточно сложные, они 2-го порядка. Но есть еще и третий обходной путь. В работе [4] показано, что поскольку матрица компонент связности Леви-Чивита кососимметричная, можно потребовать выполнения условий дуальности для этих компонент (хотя они и не образуют тензора). Тогда и тензор Римана тоже станет дуальным. Обратно, всякий дуальный тензор Римана порождается дуальной связностью Леви-Чивита при некоторой калибровке. Дуальность связности Леви-Чивита выражается более простыми дифференциальными уравнениями 1-го порядка. Недостаток этого метода лишь в том, что таким путем нельзя найти все решения уравнений дуальности тензора Римана. Приведем элементарные соображения.

В неголономном базисе, в котором положительно определенная метрика имеет канонический вид

$$
\left(\omega^{1}\right)^{2}+\left(\omega^{2}\right)^{2}+\left(\omega^{3}\right)^{2}+\left(\omega^{4}\right)^{2},
$$

дуальность матрицы связности Леви-Чивита

$$
\Omega=\left(\begin{array}{cccc}
0 & -\omega_{1}^{2} & -\omega_{1}^{3} & -\omega_{1}^{4} \\
\omega_{1}^{2} & 0 & -\omega_{2}^{3} & -\omega_{2}^{4} \\
\omega_{1}^{3} & \omega_{2}^{3} & 0 & -\omega_{3}^{4} \\
\omega_{1}^{4} & \omega_{2}^{4} & \omega_{3}^{4} & 0
\end{array}\right)
$$

выражается следующими равенствами:

$$
\omega_{1}^{2}=\epsilon \omega_{3}^{4}, \quad \omega_{1}^{3}=-\epsilon \omega_{2}^{4}, \quad \omega_{1}^{4}=\epsilon \omega_{2}^{3}, \quad \epsilon= \pm 1
$$

(автодуальность при $\epsilon=1$, антиавтодуальность при $\epsilon=-1$ ). Внешнее дифференцирование равенств (3) и формула

$$
R_{i}^{j}=d \omega_{i}^{j}+\omega_{k}^{j} \wedge \omega_{i}^{k}
$$

для 2-форм римановой кривизны приводят к равенствам

$$
R_{1}^{2}=\epsilon R_{3}^{4}, \quad R_{1}^{3}=-\epsilon R_{2}^{4}, \quad R_{1}^{4}=\epsilon R_{2}^{3}, \quad \epsilon= \pm 1,
$$

выражающим условия дуальности тензора Римана. Но если условия (3) обобщить

$$
\omega_{1}^{2}=\epsilon \omega_{3}^{4}+\alpha d f, \quad \omega_{1}^{3}=-\epsilon \omega_{2}^{4}+\beta d f, \quad \omega_{1}^{4}=\epsilon \omega_{2}^{3}+\gamma d f, \quad \epsilon= \pm 1,
$$


где $f$ - новая неизвестная функция от всех 4 переменных, а $\alpha, \beta, \gamma$ - произвольные константы, то внешнее дифференцирование равенств (5) приводит к тем же равенствам (4). Иначе говоря, решения уравнений (5) относительно неизвестных коэффициентов метрики (1) дают метрику с дуальным тензором Римана. Очевидно, решения уравнений (3) не исчерпывают всех решений системы (5) и уж тем более не исчерпывают всех решений системы (4).

Авторы статьи [7] предприняли попытку (неудачную) найти все дуальные метрики Эйнштейна. Они применили уравнения (3) к одной из метрик, считая ее (без доказательства) универсальной в классе всех положительно определенных 4-метрик. В итоге пришли к решению

$$
u^{-1}(d t+(\bar{A}, d \bar{X}))^{2}+v\left(d x^{2}+d y^{2}+d z^{2}\right)
$$

где $u$ - произвольная функция от $t, x, y$ и $z ; v$ - произвольная гармоническая функция от $x, y, z$; вектор-функция $\bar{A}=\left(A_{1}, A_{2}, A_{3}\right)$ выражается в квадратурах через $u, v$ и $\epsilon= \pm 1$. Метрика (6) дает красивые дуальные решения вакуумных уравнений Эйнштейна. Она является общим решением уравнений (3), но лишь частным решением уравнений (4). Другими словами, метрика (6) не исчерпывает всех дуальных метрик Эйнштейна.

В настоящей статье мы применяем уравнения (3) к эрмитовой метрике. Любая эрмитова метрика в комплексных переменных $z_{1}, z_{2}$ записывается в виде

$$
\psi=A d z_{1} d \bar{z}_{1}+B d z_{2} d \bar{z}_{2}+C d z_{1} d \bar{z}_{2}+\bar{C} d \bar{z}_{1} d z_{2}
$$

где $A$ и $B$ - вещественные функции, $C$ и $\bar{C}$ - комплексно сопряженные функции. Выражая $z_{1}$ и $z_{2}$ через вещественную и мнимую части, придем к вещественным переменным $t, x, y$ и $z$. С учетом положительной определенности метрика $\psi$ примет вид

$$
\psi=(a d t+u d y+v d z)^{2}+(a d x-v d y+u d z)^{2}+b^{2}\left(d y^{2}+d z^{2}\right) .
$$

Матрицу этой метрики обозначим

$$
G=\left(\begin{array}{cccc}
a^{2} & 0 & a u & a v \\
0 & a^{2} & -a v & a u \\
a u & -a v & b^{2}+u^{2}+v^{2} & 0 \\
a v & a u & 0 & b^{2}+u^{2}+v^{2}
\end{array}\right)
$$

Оператор комплексной структуры $I$, относительно которого метрика (7) инвариантна, $I^{\top} G I=G$, имеет вид

$$
I=\left(\begin{array}{cccc}
0 & 1 & 0 & 0 \\
-1 & 0 & 0 & 0 \\
0 & 0 & 0 & 1 \\
0 & 0 & -1 & 0
\end{array}\right)
$$

В первом разделе мы вычисляем компоненты связности Леви-Чивита для метрики (7) в неголономном каноническом базисе и составляем уравнения (3). Они оказываются существенно разными для $\epsilon=1$ (уравнения автодуальности) и для $\epsilon=-1$ (уравнения антиавтодуальности). Аналогичная ситуация обсуждалась в [8] для метрик с нулевой сигнатурой. 
Во втором разделе мы решаем уравнения автодуальности. Они разбиваются на три класса. Для каждого из этих классов найдены общие решения в квадратурах. В этом разделе вычисления наиболее сложные.

В третьем разделе мы упрощаем уравнения антиавтодуальности и приводим два класса частных решений в явном виде. Отметим, что наши решения не могут быть преобразованы в решение (6) статьи [7], так как в решение (6) переменные $x, y, z$ входят равноправно, а в наших решениях этого равноправия нет. Это еще раз подтверждает, что статья [7], вопреки ее заголовку, не решила проблему отыскания общего решения для (анти)автодуальных метрик Эйнштейна.

Кэлеровым многообразиям (в частности, гиперкэлеровым) посвящена обширная литература [9-16]. Известно, что риманово многообразие $(M, g)$ является гиперкэлеровым тогда и только тогда, когда на $M$ существуют две антикоммутирующие комплексные структуры I и J, каждая из которьх параллельна относительно $g$ и сохраняет $g$. В этом случае оператор

$$
x I+y J+z J I
$$

при любых вещественных $(x, y, z) \in \mathbb{R}^{3}$, для которых $x^{2}+y^{2}+z^{2}=1$, определяет параллельную комплексную структуру [1, разделы $14.10,14.11]$. В четвертом разделе для размерности 4 и метрики (7) мы устанавливаем следующее:

1) конкретный вид операторов $I$ и $J$;

2) для заданной ориентации многообразия формула (9) исчерпывает все параллельные комплексные структуры;

3) таких семейств (9) ровно два: по одному для каждой из ориентаций многообразия;

4) общий вид почти комплексной структуры, сохраняющей любую положительно определенную 4-метрику.

1. Уравнения (3) для эрмитовой метрики. Введем для эрмитовой метрики (7) неголономный канонический базис, положив

$$
\omega^{1}=a d t+u d y+v d z, \quad \omega^{2}=a d x-v d y+u d z, \quad \omega^{3}=b d y, \quad \omega^{4}=b d z .
$$

Тогда она примет вид (1). Дифференцируем внешне:

$$
\begin{gathered}
d \omega^{1}=-\frac{a_{x}}{a^{2}} \omega^{1} \wedge \omega^{2}+\left(\frac{\dot{u}}{a b}-\frac{a_{y}}{a b}-\frac{v a_{x}}{a^{2} b}\right) \omega^{1} \wedge \omega^{3}+\left(\frac{\dot{v}}{a b}-\frac{a_{z}}{a b}+\frac{u a_{x}}{a^{2} b}\right) \omega^{1} \wedge \omega^{4}+ \\
+\left(\frac{u_{x}}{a b}-\frac{u a_{x}}{a^{2} b}\right) \omega^{2} \wedge \omega^{3}+\left(\frac{v_{x}}{a b}-\frac{v a_{x}}{a^{2} b}\right) \omega^{2} \wedge \omega^{4}+ \\
+\left(\frac{u a_{z}}{a b^{2}}-\frac{v^{2} a_{x}}{a^{2} b^{2}}-\frac{u^{2} a_{x}}{a^{2} b^{2}}-\frac{v a_{y}}{a b^{2}}+\frac{v \dot{u}}{a b^{2}}+\frac{u_{x} u}{a b^{2}}-\frac{u_{z}}{b^{2}}-\frac{u \dot{v}}{a b^{2}}+\frac{v v_{x}}{a b^{2}}+\frac{v_{y}}{b^{2}}\right) \omega^{3} \wedge \omega^{4}, \\
d \omega^{2}=\frac{\dot{a}}{a^{2}} \omega^{1} \wedge \omega^{2}+\left(\frac{v \dot{a}}{a^{2} b}-\frac{\dot{v}}{a b}\right) \omega^{1} \wedge \omega^{3}+\left(\frac{\dot{u}}{a b}-\frac{u \dot{a}}{a^{2} b}\right) \omega^{1} \wedge \omega^{4}+ \\
+\left(\frac{u \dot{a}}{a^{2} b}-\frac{v_{x}}{a b}-\frac{a_{y}}{a b}\right) \omega^{2} \wedge \omega^{3}+\left(\frac{u_{x}}{a b}+\frac{v \dot{a}}{a^{2} b}-\frac{a_{z}}{a b}\right) \omega^{2} \wedge \omega^{4}+ \\
+\left(\frac{v^{2} \dot{a}}{a^{2} b^{2}}+\frac{u^{2} \dot{a}}{a^{2} b^{2}}-\frac{u a_{y}}{a b^{2}}-\frac{v a_{z}}{a b^{2}}-\frac{v \dot{v}}{a b^{2}}-\frac{v_{x} u}{a b^{2}}+\frac{v_{z}}{b^{2}}-\frac{u \dot{u}}{a b^{2}}+\frac{v u_{x}}{a b^{2}}+\frac{u_{y}}{b^{2}}\right) \omega^{3} \wedge \omega^{4},
\end{gathered}
$$




$$
\begin{aligned}
& d \omega^{3}=\frac{\dot{b}}{a b} \omega^{1} \wedge \omega^{3}+\frac{b_{x}}{a b} \omega^{2} \wedge \omega^{3}+\left(\frac{v \dot{b}}{a b^{2}}+\frac{u b_{x}}{a b^{2}}-\frac{b_{z}}{b^{2}}\right) \omega^{3} \wedge \omega^{4}, \\
& d \omega^{4}=\frac{\dot{b}}{a b} \omega^{1} \wedge \omega^{4}+\frac{b_{x}}{a b} \omega^{2} \wedge \omega^{4}+\left(\frac{v b_{x}}{a b^{2}}+\frac{b_{y}}{b^{2}}-\frac{u \dot{b}}{a b^{2}}\right) \omega^{3} \wedge \omega^{4} .
\end{aligned}
$$

Отсюда находим компоненты связности Леви-Чивита:

$$
\begin{aligned}
\omega_{1}^{2} & =-\frac{a_{x}}{a^{2}} \omega^{1}+\frac{\dot{a}}{a^{2}} \omega^{2}+\left(\frac{u a_{x}+v \dot{a}}{2 a^{2} b}-\frac{\dot{v}+u_{x}}{2 a b}\right) \omega^{3}+\left(\frac{\dot{u}-v_{x}}{2 a b}+\frac{v a_{x}-u \dot{a}}{2 a^{2} b}\right) \omega^{4}, \\
\omega_{1}^{3} & =\left(\frac{\dot{u}}{a b}-\frac{a_{y}}{a b}-\frac{v a_{x}}{a^{2} b}\right) \omega^{1}+\frac{1}{2}\left(\frac{u_{x}}{a b}-\frac{u a_{x}}{a^{2} b}+\frac{v \dot{a}}{a^{2} b}-\frac{\dot{v}}{a b}\right) \omega^{2}+\frac{\dot{b}}{a b} \omega^{3}- \\
& -\frac{1}{2}\left(\frac{u a_{z}}{a b^{2}}-\frac{v^{2} a_{x}}{a^{2} b^{2}}-\frac{u^{2} a_{x}}{a^{2} b^{2}}-\frac{v a_{y}}{a b^{2}}+\frac{v \dot{u}}{a b^{2}}+\frac{u_{x} u}{a b^{2}}-\frac{u_{z}}{b^{2}}-\frac{u \dot{v}}{a b^{2}}+\frac{v v_{x}}{a b^{2}}+\frac{v_{y}}{b^{2}}\right) \omega^{4}, \\
\omega_{1}^{4} & =\left(\frac{\dot{v}}{a b}-\frac{a_{z}}{a b}+\frac{u a_{x}}{a^{2} b}\right) \omega^{1}+\frac{1}{2}\left(\frac{v_{x}}{a b}-\frac{v a_{x}}{a^{2} b}-\frac{u \dot{a}}{a^{2} b}+\frac{\dot{u}}{a b}\right) \omega^{2}+\frac{\dot{b}}{a b} \omega^{4}+ \\
& +\frac{1}{2}\left(\frac{u a_{z}}{a b^{2}}-\frac{v^{2} a_{x}}{a^{2} b^{2}}-\frac{u^{2} a_{x}}{a^{2} b^{2}}-\frac{v a_{y}}{a b^{2}}+\frac{v \dot{u}}{a b^{2}}+\frac{u_{x} u}{a b^{2}}-\frac{u_{z}}{b^{2}}-\frac{u \dot{v}}{a b^{2}}+\frac{v v_{x}}{a b^{2}}+\frac{v_{y}}{b^{2}}\right) \omega^{3},
\end{aligned}
$$

$$
\begin{aligned}
\omega_{2}^{3} & =\frac{1}{2}\left(\frac{v \dot{a}}{a^{2} b}-\frac{\dot{v}}{a b}+\frac{u_{x}}{a b}-\frac{u a_{x}}{a^{2} b}\right) \omega^{1}+\left(\frac{u \dot{a}}{a^{2} b}-\frac{v_{x}}{a b}-\frac{a_{y}}{a b}\right) \omega^{2}+\frac{b_{x}}{a b} \omega^{3}- \\
& -\frac{1}{2}\left(\frac{v^{2} \dot{a}}{a^{2} b^{2}}+\frac{u^{2} \dot{a}}{a^{2} b^{2}}-\frac{u a_{y}}{a b^{2}}-\frac{v a_{z}}{a b^{2}}-\frac{v \dot{v}}{a b^{2}}-\frac{v_{x} u}{a b^{2}}+\frac{v_{z}}{b^{2}}-\frac{u \dot{u}}{a b^{2}}+\frac{v u_{x}}{a b^{2}}+\frac{u_{y}}{b^{2}}\right) \omega^{4},
\end{aligned}
$$

$$
\begin{aligned}
\omega_{2}^{4} & =\frac{1}{2}\left(\frac{v_{x}}{a b}-\frac{v a_{x}}{a^{2} b}-\frac{u \dot{a}}{a^{2} b}+\frac{\dot{u}}{a b}\right) \omega^{1}+\left(\frac{u_{x}}{a b}+\frac{v \dot{a}}{a^{2} b}-\frac{a_{z}}{a b}\right) \omega^{2}+\frac{b_{x}}{a b} \omega^{4}+ \\
& +\frac{1}{2}\left(\frac{v^{2} \dot{a}}{a^{2} b^{2}}+\frac{u^{2} \dot{a}}{a^{2} b^{2}}-\frac{u a_{y}}{a b^{2}}-\frac{v a_{z}}{a b^{2}}-\frac{v \dot{v}}{a b^{2}}-\frac{v_{x} u}{a b^{2}}+\frac{v_{z}}{b^{2}}-\frac{u \dot{u}}{a b^{2}}+\frac{v u_{x}}{a b^{2}}+\frac{u_{y}}{b^{2}}\right) \omega^{3},
\end{aligned}
$$

$$
\begin{array}{r}
\omega_{3}^{4}=\frac{1}{2}\left(\frac{u a_{z}}{a b^{2}}-\frac{v^{2} a_{x}}{a^{2} b^{2}}-\frac{u^{2} a_{x}}{a^{2} b^{2}}-\frac{v a_{y}}{a b^{2}}+\frac{v \dot{u}}{a b^{2}}+\frac{u_{x} u}{a b^{2}}-\frac{u_{z}}{b^{2}}-\frac{u \dot{v}}{a b^{2}}+\frac{v v_{x}}{a b^{2}}+\frac{v_{y}}{b^{2}}\right) \omega^{1}+ \\
+\frac{1}{2}\left(\frac{v^{2} \dot{a}}{a^{2} b^{2}}+\frac{u^{2} \dot{a}}{a^{2} b^{2}}-\frac{u a_{y}}{a b^{2}}-\frac{v a_{z}}{a b^{2}}-\frac{v \dot{v}}{a b^{2}}-\frac{v_{x} u}{a b^{2}}+\frac{v_{z}}{b^{2}}-\frac{u \dot{u}}{a b^{2}}+\frac{v u_{x}}{a b^{2}}+\frac{u_{y}}{b^{2}}\right) \omega^{2}+ \\
+\left(\frac{v \dot{b}}{a b^{2}}+\frac{u b_{x}}{a b^{2}}-\frac{b_{z}}{b^{2}}\right) \omega^{3}+\left(\frac{v b_{x}}{a b^{2}}+\frac{b_{y}}{b^{2}}-\frac{u \dot{b}}{a b^{2}}\right) \omega^{4}
\end{array}
$$


Уравнения дуальности (3) принимают следующий вид:

$$
\begin{aligned}
& \frac{u a_{z}}{a b^{2}}-\frac{v^{2} a_{x}}{a^{2} b^{2}}-\frac{u^{2} a_{x}}{a^{2} b^{2}}-\frac{v a_{y}}{a b^{2}}+\frac{v \dot{u}}{a b^{2}}+\frac{u_{x} u}{a b^{2}}-\frac{u_{z}}{b^{2}}-\frac{u \dot{v}}{a b^{2}}+\frac{v v_{x}}{a b^{2}}+\frac{v_{y}}{b^{2}}+\frac{2 \epsilon a_{x}}{a^{2}}=0, \\
& \frac{v^{2} \dot{a}}{a^{2} b^{2}}+\frac{u^{2} \dot{a}}{a^{2} b^{2}}-\frac{u a_{y}}{a b^{2}}-\frac{v a_{z}}{a b^{2}}-\frac{v \dot{v}}{a b^{2}}-\frac{v_{x} u}{a b^{2}}+\frac{v_{z}}{b^{2}}-\frac{u \dot{u}}{a b^{2}}+\frac{v u_{x}}{a b^{2}}+\frac{u_{y}}{b^{2}}-\frac{2 \epsilon \dot{a}}{a^{2}}=0, \\
& 2\left(\frac{b_{z}}{b}-\frac{v \dot{b}}{a b}-\frac{u b_{x}}{a b}\right)+\epsilon\left(\frac{v \dot{a}}{a^{2}}-\frac{\dot{v}}{a}-\frac{u_{x}}{a}+\frac{u a_{x}}{a^{2}}\right)=0, \\
& 2\left(\frac{u \dot{b}}{a b}-\frac{v b_{x}}{a b}-\frac{b_{y}}{b}\right)+\epsilon\left(\frac{\dot{u}}{a}-\frac{u \dot{a}}{a^{2}}-\frac{v_{x}}{a}+\frac{v a_{x}}{a^{2}}\right)=0, \\
& 2\left(\frac{\dot{u}}{a}-\frac{a_{y}}{a}-\frac{v a_{x}}{a^{2}}\right)+\epsilon\left(\frac{v_{x}}{a}-\frac{v a_{x}}{a^{2}}-\frac{u \dot{a}}{a^{2}}+\frac{\dot{u}}{a}\right)=0, \\
& 2\left(\frac{u_{x}}{a}+\frac{v \dot{a}}{a^{2}}-\frac{a_{z}}{a}\right)+\epsilon\left(\frac{u_{x}}{a}-\frac{u a_{x}}{a^{2}}+\frac{v \dot{a}}{a^{2}}-\frac{\dot{v}}{a}\right)=0, \\
& 2\left(\frac{a_{z}}{a}-\frac{\dot{v}}{a}-\frac{u a_{x}}{a^{2}}\right)+\epsilon\left(\frac{u_{x}}{a}-\frac{u a_{x}}{a^{2}}+\frac{v \dot{a}}{a^{2}}-\frac{\dot{v}}{a}\right)=0, \\
& 2\left(\frac{v_{x}}{a}+\frac{a_{y}}{a}-\frac{u \dot{a}}{a^{2}}\right)+\epsilon\left(\frac{v_{x}}{a}-\frac{v a_{x}}{a^{2}}-\frac{u \dot{a}}{a^{2}}+\frac{\dot{u}}{a}\right)=0 .
\end{aligned}
$$

Вычтем $(11)_{1}$ и $(11)_{8}$, а также $(11)_{2}$ и $(11)_{7}$ :

$$
\frac{a_{x}}{a}+\frac{b_{x}}{b}=0, \quad \frac{\dot{a}}{a}+\frac{\dot{b}}{b}=0 .
$$

Теперь вычтем $(11)_{6}$ и $(11)_{9}$, а также $(11)_{5}$ и $(11)_{10}$ :

$$
\frac{u_{x}}{a}+\frac{u a_{x}}{a^{2}}+\frac{v \dot{a}}{a^{2}}+\frac{\dot{v}}{a}=\frac{2 a_{z}}{a}, \quad \frac{\dot{u}}{a}+\frac{u \dot{a}}{a^{2}}-\frac{v a_{x}}{a^{2}}-\frac{v_{x}}{a}=\frac{2 a_{y}}{a} .
$$

Сложим (11)6 и $(11)_{9}$, а также $(11)_{5}$ и $(11)_{10}$ :

$$
(\epsilon+1)\left(\frac{u_{x}}{a}-\frac{u a_{x}}{a^{2}}+\frac{v \dot{a}}{a^{2}}-\frac{\dot{v}}{a}\right)=0, \quad(\epsilon+1)\left(\frac{v_{x}}{a}-\frac{v a_{x}}{a^{2}}+\frac{\dot{u}}{a}-\frac{u \dot{a}}{a^{2}}\right)=0 .
$$

Запишем уравнения $(11)_{3,4}$, упростив их с помощью (13)

$\frac{b_{z}}{b}-\frac{v \dot{b}}{a b}-\frac{u b_{x}}{a b}+\epsilon\left(\frac{v \dot{a}}{a^{2}}+\frac{u a_{x}}{a^{2}}-\frac{a_{z}}{a}\right)=0, \quad \frac{u \dot{b}}{a b}-\frac{v b_{x}}{a b}-\frac{b_{y}}{b}+\epsilon\left(\frac{a_{y}}{a}-\frac{u \dot{a}}{a^{2}}+\frac{v a_{x}}{a^{2}}\right)=0$.

Исключим из этих уравнений $b_{x} / b$ и $\dot{b} / b$ с помощью $(12)$ :

$$
\frac{b_{z}}{b}-\frac{\varepsilon a_{z}}{a}+(\epsilon+1)\left(\frac{v \dot{a}}{a^{2}}+\frac{u a_{x}}{a^{2}}\right)=0, \quad \frac{b_{y}}{b}-\frac{\varepsilon a_{y}}{a}+(\epsilon+1)\left(\frac{u \dot{a}}{a^{2}}-\frac{v a_{x}}{a^{2}}\right)=0 .
$$

Итак, система (11) из 10 уравнений равносильна 10 уравнениям $(11)_{1,2}$, (12), (13), (14) и (15). 
2. Автодуальный случай, $\boldsymbol{\epsilon}=\mathbf{1}$. Уравнения (14) примут вид

$$
\frac{u_{x}}{a}-\frac{u a_{x}}{a^{2}}+\frac{v \dot{a}}{a^{2}}-\frac{\dot{v}}{a}=0, \quad \frac{v_{x}}{a}-\frac{v a_{x}}{a^{2}}+\frac{\dot{u}}{a}-\frac{u \dot{a}}{a^{2}}=0 .
$$

Уравнения (12) равносильны

$$
a b=C(y, z),
$$

откуда

$$
\frac{b_{y}}{b}=\frac{C_{y}}{C}-\frac{a_{y}}{a}, \quad \frac{b_{z}}{b}=\frac{C_{z}}{C}-\frac{a_{z}}{a} .
$$

С учетом этого, уравнения (15) примут вид

$$
\frac{C_{z}}{C}-\frac{2 a_{z}}{a}+2\left(\frac{v \dot{a}}{a^{2}}+\frac{u a_{x}}{a^{2}}\right)=0, \quad \frac{C_{y}}{C}-\frac{2 a_{y}}{a}+2\left(\frac{u \dot{a}}{a^{2}}-\frac{v a_{x}}{a^{2}}\right)=0 .
$$

Подставим сюда выражения из (13):

$$
\frac{C_{z}}{C}-\frac{u_{x}}{a}-\frac{\dot{v}}{a}+\frac{v \dot{a}}{a^{2}}+\frac{u a_{x}}{a^{2}}=0, \quad \frac{C_{y}}{C}-\frac{\dot{u}}{a}+\frac{v_{x}}{a}+\frac{u \dot{a}}{a^{2}}-\frac{v a_{x}}{a^{2}}=0
$$

и еще упростим с помощью (16):

$$
\dot{v}=\frac{v \dot{a}}{a}+\frac{a C_{z}}{2 C}, \quad v_{x}=\frac{v a_{x}}{a}-\frac{a C_{y}}{2 C} .
$$

Сами уравнения (16) после исключения из них $\dot{v}$ и $v_{x}$ позволяют выразить

$$
\dot{u}=\frac{u \dot{a}}{a}+\frac{a C_{y}}{2 C}, \quad u_{x}=\frac{u a_{x}}{a}+\frac{a C_{z}}{2 C} .
$$

С учетом (17) уравнения (11) 1,2 перепишем в виде

$$
\begin{gathered}
u a_{z}-\left(u^{2}+v^{2}\right) \frac{a_{x}}{a}-v a_{y}+v \dot{u}+u_{x} u-a u_{z}-u \dot{v}+v v_{x}+a v_{y}+2 C^{2} \frac{a_{x}}{a^{3}}=0 \\
\left(u^{2}+v^{2}\right) \frac{\dot{a}}{a}-u a_{y}-v a_{z}-v \dot{v}-v_{x} u+a v_{z}-u \dot{u}+v u_{x}+a u_{y}-2 C^{2} \frac{\dot{a}}{a^{3}}=0 .
\end{gathered}
$$

После подстановки в них выражений из (18) и (19) они упрощаются:

$$
u a_{z}-a u_{z}+a v_{y}-v a_{y}+2 C^{2} \frac{a_{x}}{a^{3}}=0, \quad a v_{z}-v a_{z}+a u_{y}-u a_{y}-2 C^{2} \frac{\dot{a}}{a^{3}}=0 .
$$

Уравнения (13) после исключения из них $\dot{v}, v_{x}, \dot{u}$ и $u_{x}$ с помощью (18) и (19) дают

$$
a_{y}=\frac{u \dot{a}}{a}+\frac{a C_{y}}{2 C}-\frac{v a_{x}}{a}, \quad a_{z}=\frac{u a_{x}}{a}+\frac{a C_{z}}{2 C}+\frac{v \dot{a}}{a} .
$$

Положим

$$
U \equiv \frac{u}{a}, \quad V \equiv \frac{v}{a}, \quad C \equiv e^{P},
$$


где $P$ зависит только от $y$ и $z$. Тогда (18) и (19) принимают вид

$$
\dot{U}=\frac{P_{y}}{2}, \quad U_{x}=\frac{P_{z}}{2}, \quad \dot{V}=\frac{P_{z}}{2}, \quad V_{x}=-\frac{P_{y}}{2} .
$$

Отсюда, интегрируя, получаем

$$
U=\frac{1}{2} P_{y} t+\frac{1}{2} P_{z} x+D(y, z), \quad V=\frac{1}{2} P_{z} t-\frac{1}{2} P_{y} x+E(y, z) .
$$

Уравнения (20) после обозначений (22) записываются в виде

$$
V_{z}+U_{y}=-\frac{1}{2} e^{2 P}\left(a^{-4}\right)^{\cdot}, \quad V_{y}-U_{z}=\frac{1}{2} e^{2 P}\left(a^{-4}\right)_{x}
$$

Подставим сюда $U$ и $V$ из (23):

$$
\left(a^{-4}\right)_{x}=\frac{2\left(E_{y}-D_{z}\right)-x \Delta P}{e^{2 P}}, \quad\left(a^{-4}\right)^{\cdot}=\frac{-2\left(E_{z}+D_{y}\right)-t \Delta P}{e^{2 P}}
$$

где $\Delta P \equiv P_{y y}+P_{z z}$ - лапласиан функции $P(y, z)$. Интегрируя, получаем

$$
a^{-4}=e^{-2 P}\left(F(y, z)-\frac{1}{2}\left(t^{2}+x^{2}\right) \Delta P-2\left(E_{z}+D_{y}\right) t+2\left(E_{y}-D_{z}\right) x\right)
$$

где $P, D, E$ и $F-$ функции только от $y$ и $z$.

Уравнения (21) после обозначений (22) можно записать в виде

$$
\left(a^{-4}\right)_{y}=U\left(a^{-4}\right)^{\cdot}-V\left(a^{-4}\right)_{x}-2 a^{-4} P_{y}, \quad\left(a^{-4}\right)_{z}=U\left(a^{-4}\right)_{x}+V\left(a^{-4}\right)^{\cdot}-2 a^{-4} P_{z} .
$$

Вычисляя левые части с помощью (25), а правые - с помощью (23), (24) и $(25)$, получим систему из 8 дифференциальных уравнений на 4 функции $P$, $D, E$ и $F$ от переменных $y$ и $z$ :

$$
\begin{gathered}
\Delta P_{y}=P_{y} \Delta P, \quad \Delta P_{z}=P_{z} \Delta P, \quad \Delta D=D \Delta P, \quad \Delta E=E \Delta P \\
2 E_{y z}=D_{z z}-D_{y y}+P_{y} D_{y}-P_{z} D_{z}+P_{y} E_{z}+P_{z} E_{y} \\
2 D_{y z}=E_{y y}-E_{z z}+P_{y} D_{z}+P_{z} D_{y}-P_{y} E_{y}+P_{z} E_{z} \\
F_{y}=2 E D_{z}-2 D D_{y}-2 D E_{z}-2 E E_{y}, \quad F_{z}=2 D E_{y}-2 D D_{z}-2 E D_{y}-2 E E_{z}
\end{gathered}
$$

знак $\Delta$ - лапласиан от двух переменных $y$ и $z$.

Уравнения (26) запишем в виде

$$
\Delta P+\alpha e^{P}=0, \quad \Delta D+\alpha D e^{P}=0, \quad \Delta E+\alpha E e^{P}=0,
$$

где $\alpha$ - неотрицательная константа. Уравнения (27) преобразуем так:

$$
\left(\frac{D_{y}+E_{z}}{e^{P}}\right)_{y}=\left(\frac{D_{z}-E_{y}}{e^{P}}\right)_{z}, \quad\left(\frac{D_{y}+E_{z}}{e^{P}}\right)_{z}=-\left(\frac{D_{z}-E_{y}}{e^{P}}\right)_{y} .
$$


Это означает, что функции $Q \equiv e^{-P}\left(D_{y}+E_{z}\right)$ и $S \equiv e^{-P}\left(D_{z}-E_{y}\right)$ являются вещественной и мнимой частями комплексной голоморфной функции, то есть являются сопряженными гармоническими функциями от $y$ и z. Отсюда

$$
D_{y}+E_{z}=Q e^{P}, \quad D_{z}-E_{y}=S e^{P}
$$

Записывая условия интегрируемости для функций $D$ и $E$ с помощью (29), получаем

$$
\alpha E=S_{y}+S P_{y}-Q_{z}-Q P_{z}, \quad-\alpha D=S_{z}+S P_{z}+Q_{y}+Q P_{y}
$$

Далее рассмотрим два случая.

I случай, $\boldsymbol{\alpha} \neq \mathbf{0}$. Тогда функции $D$ и $E$ выражаются через $P, Q$ и $S$ из (31), и легко проверить, что они обращают в тождество $(29)_{2,3}$. Непосредственной подстановкой можно убедиться, что функция

$$
F=\beta-\frac{2}{\alpha}\left(Q^{2}+S^{2}\right) e^{P}, \quad \beta=\mathrm{const}
$$

является общим решением уравнений (28).

Итак, общее решение уравнений автодуальности при $\alpha \neq 0$ зависит от пары сопряженных гармонических функций $Q(y, z), S(y, z)$, двух констант $\alpha, \beta$ и функции $P(y, z)$, удовлетворяющей уравнению $(29)_{1}$. При этом из (25) и (17) получаем

$$
a^{-4}=e^{-P}\left(\beta e^{-P}-\frac{2}{\alpha}\left(Q^{2}+S^{2}\right)+\frac{\alpha}{2}\left(t^{2}+x^{2}\right)-2 Q t-2 S x\right), \quad b=\frac{e^{P}}{a},
$$

а из (23) и $(31)-$

$$
\begin{aligned}
& u=\left(\frac{1}{2}\left(P_{y} t+P_{z} x\right)-\frac{1}{\alpha}\left(2 S_{z}+S P_{z}+Q P_{y}\right)\right) a, \\
& v=\left(\frac{1}{2}\left(P_{z} t-P_{y} x\right)+\frac{1}{\alpha}\left(2 S_{y}+S P_{y}-Q P_{z}\right)\right) a .
\end{aligned}
$$

II случай, $\boldsymbol{\alpha}=\mathbf{0}$. Тогда уравнения (29) означают, что $\Delta P=\Delta D=$ $=\Delta E=0$. Равенства (31) с учетом того, что $Q_{z}=-S_{y}$ и $Q_{y}=S_{z}$, можно записать в виде

$$
2 S_{y}=Q P_{z}-S P_{y}, \quad 2 Q_{y}=-S P_{z}-Q P_{y}
$$

Умножим первое из них на $S$, второе - на $Q$ и сложим:

$$
\left(S^{2}+Q^{2}\right)_{y}=-\left(S^{2}+Q^{2}\right) P_{y}
$$

Аналогичными манипуляциями из (31) можно получить

$$
\left(S^{2}+Q^{2}\right)_{z}=-\left(S^{2}+Q^{2}\right) P_{z}
$$

Отсюда

$$
S^{2}+Q^{2}=\beta e^{-P}, \quad \beta=\text { const } \geqslant 0 .
$$


Это общее решение уравнений (31) при $\alpha=0$. Здесь снова возможны два случая.

Случай $\mathbf{I I}_{\mathbf{1}}, \boldsymbol{\beta}=\boldsymbol{S}=\boldsymbol{Q}=\mathbf{0}$. Тогда формулы (30) перепишутся в виде

$$
D_{y}+E_{z}=0, \quad D_{z}-E_{y}=0 .
$$

Это означает, что $D$ и $E$ - пара сопряженных гармонических функций. В силу (33) правые части уравнений (28) равны нулю. Следовательно, $F=$ const. Из (25) видно, что $F$ может быть только положительной. Удобно положить $F=\gamma^{-4}$. Тогда из (25) $a=\gamma e^{P / 2}$, а из (17) $b=\gamma^{-1} e^{P / 2}$. Наконец, из (22) и (23) получаем

$$
u=\gamma e^{P / 2}\left(\frac{1}{2} P_{y} t+\frac{1}{2} P_{z} x+D\right), \quad v=\gamma e^{P / 2}\left(\frac{1}{2} P_{z} t-\frac{1}{2} P_{y} x+E\right) .
$$

Произвол решения случая $\mathrm{II}_{1}$ почти такой же, как и в случае I: $P(y, z)-$ произвольная гармоническая функция, $D(y, z)$ и $E(y, z)$ - сопряженные гармонические функции и одна константа $\gamma>0$. Однако тензор Римана для случая $\mathrm{II}_{1}$ нулевой, то есть эрмитова метрика (7) - плоская. При нулевом тензоре Римана автодуальность не отличается от антиавтодуальности.

Случай $\mathbf{I I}_{2}, \boldsymbol{\beta}>\mathbf{0}$. Из (32) и (30) можно явно выразить гармонические функции $P, Q$ и $S$ через гармонические функции $D$ и $E$ :

$$
e^{P}=\frac{\left(D_{y}+E_{z}\right)^{2}+\left(D_{z}-E_{y}\right)^{2}}{\beta}, \quad Q=e^{-P}\left(D_{y}+E_{z}\right), \quad S=e^{-P}\left(D_{z}-E_{y}\right),
$$

причем из этих формул и из гармоничности $D$ и $E$ вытекает гармоничность $P, Q$ и $S$. Функция $F$ тоже выражается через $D$ и $E$ из формул $(28)$ одной квадратурой с точностью до константы интегрирования $\gamma$. Таким образом, в случае $\mathrm{II}_{2}$ произвол решения определяется двумя не связанными между собой несопряженными гармоническими функциями $D$ и $E$ и двумя константами $\beta$ и $\gamma$, а само решение использует лишь одну квадратуру. Из (25) и (17) вычисляются $a$ и $b$ :

$$
a^{-4}=\frac{\beta^{2}\left(F-2\left(E_{z}+D_{y}\right) t+2\left(E_{y}-D_{z}\right) x\right)}{\left(\left(D_{y}+E_{z}\right)^{2}+\left(D_{z}-E_{y}\right)^{2}\right)^{2}}, \quad b=\frac{\left(D_{y}+E_{z}\right)^{2}+\left(D_{z}-E_{y}\right)^{2}}{\beta a} .
$$

Из (23) получаются $u$ и $v$ :

$$
u=\left(\frac{1}{2} P_{y} t+\frac{1}{2} P_{z} x+D\right) a, \quad v=\left(\frac{1}{2} P_{z} t-\frac{1}{2} P_{y} x+E\right) a .
$$

3. Антиавтодуальный случай, $\boldsymbol{\epsilon}=\mathbf{- 1}$. Уравнения (14) выполняются тождественно, а уравнения (15) дают

$$
\frac{b_{z}}{b}+\frac{a_{z}}{a}=0, \quad \frac{b_{y}}{b}+\frac{a_{y}}{a}=0 .
$$

Вместе с (12) это эквивалентно $a b=C=$ const. Перенормировкой переменных $y$ и $z$ можно добиться равенства $C=1$, так что считаем $b=a^{-1}$. Остались 4 уравнения: (13) и (11) 1,2 . Положим

$$
u a \equiv U, \quad v a \equiv V, \quad a^{2} \equiv A .
$$


Тогда уравнения (13) принимают вид

$$
U_{x}+\dot{V}=A_{z}, \quad \dot{U}-V_{x}=A_{y}
$$

a $(11)_{1,2}$ становятся такими:

$$
U_{y}+V_{z}=\left(\frac{V^{2}+U^{2}+1}{A}\right), \quad U_{z}-V_{y}=\left(\frac{V^{2}+U^{2}+1}{A}\right)_{x} .
$$

Итак, все уравнения антиавтодуальности свелись к 4 уравнениям в частных производных 1-го порядка (34) и (35) на 3 функции: $U, V$ и $A$ от всех четырех переменных. Можно исключить правые части как из уравнений (34), так и из уравнений (35). Это приводит к одному и тому же условию

$$
U_{x y}-\dot{U}_{z}+\dot{V}_{y}+V_{x z}=0
$$

Нам не удалось найти общее решение системы (34), (35). Но можно получить частное решение в элементарных функциях, если наложить дополнительное условие $A=U$. Тогда уравнения (34) и (35) запишутся в виде

$$
\begin{gathered}
V_{x}=\dot{U}-U_{y}, \quad \dot{V}=U_{z}-U_{x} \\
V_{y}=U_{z}-U_{x}+\frac{2 V\left(U_{y}-\dot{U}\right)}{U}+\frac{U_{x}\left(V^{2}+1\right)}{U^{2}}, \\
V_{z}=\dot{U}-U_{y}+\frac{2 V\left(U_{z}-U_{x}\right)}{U}-\frac{\dot{U}\left(V^{2}+1\right)}{U^{2}} .
\end{gathered}
$$

Система уравнений (36)-(38) все еще достаточно сложная. Но структура этих уравнений подсказывает, какие необходимо еще ввести ограничения, чтобы получить легко интегрируемые уравнения. Положим дополнительно

$$
U_{y}-\dot{U}=0, \quad U_{z}-U_{x}=0 .
$$

Тогда в силу (36) функция $V$ не будет зависеть от $t$ и $x$, а уравнения (37) и (38) запишутся в виде

$$
\frac{V_{y}}{V^{2}+1}=\frac{U_{x}}{U^{2}}, \quad \frac{V_{z}}{V^{2}+1}=-\frac{\dot{U}}{U^{2}}
$$

Так как левые части не зависят от $t$ и $x$, находим

$$
U=\frac{V^{2}+1}{t V_{z}-x V_{y}+f}
$$

где $f(y, z)$ - произвольная функция. Подставим это в уравнения (39) и получим 5 уравнений:

$$
\begin{gathered}
V_{y z}\left(V^{2}+1\right)=2 V V_{y} V_{z}, \quad 2 V V_{y}^{2}=V_{y y}\left(V^{2}+1\right), \quad 2 V V_{z}^{2}=V_{z z}\left(V^{2}+1\right) \\
\left(V^{2}+1\right)\left(f_{y}-V_{z}\right)=2 V V_{y} f, \quad\left(V^{2}+1\right)\left(f_{z}+V_{y}\right)=2 V V_{z} f
\end{gathered}
$$


Из (40) 1,2,3 сначала находим

$$
V_{y}=C_{1}\left(V^{2}+1\right), \quad V_{z}=C_{2}\left(V^{2}+1\right), \quad C_{1}, C_{2}=\text { const },
$$

а затем и

$$
V=\operatorname{tg}\left(C_{1} y+C_{2} z+C_{3}\right), \quad C_{1}, C_{2}, C_{3}=\text { const. }
$$

Оставшиеся два уравнения $(40)_{4,5}$ позволяют найти

$$
f=\frac{C_{2} y-C_{1} z+C_{4}}{\cos ^{2}\left(C_{1} y+C_{2} z+C_{3}\right)}, \quad C_{1}, C_{2}, C_{3}, C_{4}=\text { const. }
$$

Параметры метрики (7) таковы:

$$
\begin{aligned}
& a=u=\frac{1}{\sqrt{C_{2}(t+y)-C_{1}(x+z)+C_{4}}}, \quad b=a^{-1}, \\
& v=a^{-1} \operatorname{tg}\left(C_{1} y+C_{2} z+C_{3}\right), \quad C_{1}, C_{2}, C_{3}, C_{4}=\text { const. }
\end{aligned}
$$

Другое частное решение системы уравнений (34), (35) получается при

$$
V=\gamma U, \quad U_{x}=\dot{U}=0, \quad \gamma=\text { const. }
$$

Решение для этого случая таково:

$$
\begin{gathered}
a=\frac{1}{\sqrt{C_{1} t+C_{2} x+C_{3}}}, \quad b=a^{-1}, \quad v=\gamma u \\
u=\frac{1}{a \sqrt{1+\gamma^{2}}} \operatorname{tg} \frac{\left(C_{1}-C_{2} \gamma\right) y+\left(C_{1} \gamma+C_{2}\right) z+C_{4}}{\sqrt{1+\gamma^{2}}}, \quad C_{1}, C_{2}, C_{3}, C_{4}=\text { const. }
\end{gathered}
$$

4. Связь с кэлеровыми метриками. Известно, что в размерности 4 многообразие гиперкэлерово тогда и только тогда, когда оно эйнштейново и антиавтодуально [2, гл. 9]. С другой стороны, справедливо следующее утверждение: риманово 4-многообразие гиперкэлерово тогда и только тогда, когда оно кэлерово и Риччи-плоско [1, разд. 14.22]. Следовательно, все наши антиавтодуальные решения дают гиперкэлерову метрику, а автодуальные решения дают кэлерову метрику только в плоском случае, то есть это решения $\mathrm{II}_{1}$.

В данном разделе мы найдем явный вид всех параллельных относительно метрики (7) комплексных структур, сохраняющих эту метрику (в случае дуальности связности Леви-Чивита). Во введении мы отмечали, что комплексная структура (8) сохраняет метрику (7). Найдем условие ее параллельности. Матрица (8) задана в голономном базисе $(d t, d x, d y, d z)$, а 1-формы связности Леви-Чивита подсчитаны в неголономном базисе (1). Из (10) видно, что матрица перехода от голономного базиса к неголономному такова:

$$
P=\left(\begin{array}{cccc}
a & 0 & u & v \\
0 & a & -v & u \\
0 & 0 & b & 0 \\
0 & 0 & 0 & b
\end{array}\right)
$$


Оказывается, что в неголономном базисе матрица (8) сохраняет свой вид:

$$
P I P^{-1}=I \text {. }
$$

Чтобы метрика (7) была кэлеровой, оператор $I$ должен быть параллелен относительно метрики (7), то есть его ковариантный дифференциал должен быть равен нулю $(D I=0)$. Ввиду постоянства компонент матрицы $I$ это равенство равносильно перестановочности матрицы $I$ и матрицы связности Леви-Чивита (2) $\Omega$ :

$$
I \Omega=\Omega I
$$

Последнее равенство равносильно равенствам

$$
\omega_{1}^{3}=\omega_{2}^{4}, \quad \omega_{1}^{4}=-\omega_{2}^{3} .
$$

Сравнивая эти равенства с условиями дуальности (3) связности, заключаем, что оператор $I$ параллелен в антиавтодуальном случае (при $\epsilon=-1)$.

Если почти комплексная структура $C$, заданная в неголономном базисе $\omega^{1}, \omega^{2}, \omega^{3}, \omega^{4}$, сохраняет квадратичную форму (1), то должны выполняться два условия:

$$
C^{2}=-E, \quad C^{\top} C=E,
$$

где $E$ - единичная матрица 4-го порядка. Отсюда $C^{\top}=-C$. Иначе говоря, матрица $C$ ортогональная и кососимметричная. Запишем подробно условия ортогональности кососимметричной матрицы $C=\left(c_{i j}\right)$ :

$$
\begin{aligned}
\left(c_{12}\right)^{2}+\left(c_{13}\right)^{2}+\left(c_{14}\right)^{2} & =1, & & \left(c_{12}\right)^{2}+\left(c_{23}\right)^{2}+\left(c_{24}\right)^{2}=1, \\
\left(c_{13}\right)^{2}+\left(c_{23}\right)^{2}+\left(c_{34}\right)^{2} & =1, & & \left(c_{14}\right)^{2}+\left(c_{24}\right)^{2}+\left(c_{34}\right)^{2}=1, \\
c_{13} c_{23}+c_{14} c_{24} & =0, & & c_{12} c_{23}-c_{14} c_{34}=0, \\
c_{12} c_{24}+c_{13} c_{34} & =0, & & c_{12} c_{13}+c_{24} c_{34}=0, \\
c_{12} c_{14}-c_{23} c_{34} & =0, & & c_{13} c_{14}+c_{23} c_{24}=0 .
\end{aligned}
$$

Предполагая, что $c_{14}$ и $c_{23}$ не равны нулю, исключим $c_{12}$ из уравнений $(43)_{6,9}$ :

$$
c_{34}\left(\left(c_{14}\right)^{2}-\left(c_{23}\right)^{2}\right)=0 .
$$

Отсюда возможны три случая:

1) $c_{14}=c_{23}$; остальные равенства (43) дают

$$
\left(c_{12}\right)^{2}+\left(c_{13}\right)^{2}+\left(c_{14}\right)^{2}=1, \quad c_{13}=-c_{24}, \quad c_{12}=c_{34}
$$

2) $c_{14}=-c_{23}$; остальные равенства (43) сводятся к

$$
\left(c_{12}\right)^{2}+\left(c_{13}\right)^{2}+\left(c_{14}\right)^{2}=1, \quad c_{13}=c_{24}, \quad c_{12}=-c_{34}
$$

3) $c_{34}=0$; в этом случае условия (43) приводят либо к случаю 1), либо к случаю 2), поэтому случай 3) не дает новых решений системы (43). 
Мы получили решения (44) и (45) в предположении, что $c_{14}$ и $c_{23}$ не равны нулю. Легко убедиться, что и при равенстве нулю $c_{14}$ или $c_{23}$ формулы (44) и (45) остаются верными, то есть они дают общее решение системы (43).

В случае 1) в силу (44) имеем

$$
C=c_{12} I+c_{13} J+c_{14} K, \quad\left(c_{12}\right)^{2}+\left(c_{13}\right)^{2}+\left(c_{14}\right)^{2}=1,
$$

где

$$
J=\left(\begin{array}{cccc}
0 & 0 & 1 & 0 \\
0 & 0 & 0 & -1 \\
-1 & 0 & 0 & 0 \\
0 & 1 & 0 & 0
\end{array}\right), \quad K=J I=\left(\begin{array}{cccc}
0 & 0 & 0 & 1 \\
0 & 0 & 1 & 0 \\
0 & -1 & 0 & 0 \\
-1 & 0 & 0 & 0
\end{array}\right)
$$

В случае 2) в силу (45) получаем

$$
C=c_{12} N+c_{13} M+c_{14} Q, \quad\left(c_{12}\right)^{2}+\left(c_{13}\right)^{2}+\left(c_{14}\right)^{2}=1
$$

где

$$
\begin{gathered}
N=\left(\begin{array}{cccc}
0 & 1 & 0 & 0 \\
-1 & 0 & 0 & 0 \\
0 & 0 & 0 & -1 \\
0 & 0 & 1 & 0
\end{array}\right), \quad M=\left(\begin{array}{ccccc}
0 & 0 & 1 & 0 \\
0 & 0 & 0 & 1 \\
-1 & 0 & 0 & 0 \\
0 & -1 & 0 & 0
\end{array}\right) \\
Q=N M=\left(\begin{array}{cccc}
0 & 0 & 0 & 1 \\
0 & 0 & -1 & 0 \\
0 & 1 & 0 & 0 \\
-1 & 0 & 0 & 0
\end{array}\right) .
\end{gathered}
$$

Условие параллельности (42) операторов $J$ и $K$ сводится к равенствам

$$
\omega_{1}^{4}=-\omega_{2}^{3}, \quad \omega_{1}^{3}=\omega_{2}^{4}, \quad \omega_{1}^{2}=-\omega_{3}^{4} .
$$

Эти равенства выполняются в силу условий антиавтодуальности (3) (при $\epsilon=-1)$. Чтобы оператор $C$, заданный формулой (46), был параллелен, необходимо и достаточно, чтобы коэффициенты $c_{12}, c_{13}, c_{14}$ были константами.

Операторы $M, N$ и $Q$ параллельны только при выполнении условий автодуальности (3) (при $\epsilon=1)$. Чтобы оператор $C$, заданный формулой $(47)$, был параллелен, необходимо и достаточно, чтобы коэффициенты $c_{12}, c_{13}, c_{14}$ были константами.

Возвращение оператора $C$ к голономному базису по формуле $P^{-1} C P$ дает операторы почти комплексной структуры в голономном базисе, сохраняющие метрику (7) и параллельные относительно этой метрики как в случае (46), так и в случае (47). Однако для автодуального случая операторов (47) это дает противоречие с утверждением, сформулированным в начале настоящего раздела, которое требует эйнштейновости и антиавтодуальности.

Для снятия этого противоречия рассмотрим оператор $T$ перестановки элементов $\omega^{2}$ и $\omega^{3}$ неголономного канонического базиса. Он имеет вид

$$
T=\left(\begin{array}{llll}
1 & 0 & 0 & 0 \\
0 & 0 & 1 & 0 \\
0 & 1 & 0 & 0 \\
0 & 0 & 0 & 1
\end{array}\right), \quad T^{2}=E
$$


Легко проверить справедливость формул

$$
T N T=J, \quad T M T=I, \quad T Q T=K .
$$

Следовательно, операторы (47) переходят в (46) и наоборот. Перестановка двух элементов базиса меняет ориентацию многообразия (но не меняет квадратичную форму (1)), а смена ориентации меняет знак оператора Ходжа (см. [8]). При этом понятия автодуальности и антиавтодуальности меняются местами. Заметим, что определитель матрицы перехода (41) от голономного базиса к неголономному положительный, поэтому виды дуальности в базисах $\omega^{1}, \omega^{2}, \omega^{3}, \omega^{4}$ и $d t, d x, d y, d z$ совпадают.

Итак, (46) - это общий вид операторов почти комплексной структуры, параллельных и сохраняющих метрику (7), для одной ориентации многообразия, а формула (47) - для другой ориентации.

На самом деле начальное утверждение этого раздела по умолчанию предполагает следующую формулировку: в размерности 4 многообразие с заданной ориентацией гиперкэлерово тогда и только тогда, когда оно эйнштейново и антиавтодуально. В этом случае никаких противоречий с нашими результатами нет. Из известного утверждения, сформулированного в конце введения, следует, что все операторы (46) и (47) - без кручения.

Приведенный в данном разделе анализ показывает, что формулы (46) и (47) дают (в неголономном каноническом базисе) все почти комплексные структуры, сохраняющие произвольную положительно определенную метрику (1). Однако у произвольной метрики коэффициенты $c_{12}, c_{13}$ и $c_{14}$ будут не константами, а функциями от точки многообразия с условием $\left(c_{12}\right)^{2}+$ $+\left(c_{13}\right)^{2}+\left(c_{14}\right)^{2}=1$. Среди этих структур может не найтись структур с нулевым кручением. Поэтому такая метрика не обязана быть эрмитовой. Тем не менее всякую положительно определенную метрику можно считать почти эрмитовой.

Заключение. В данной статье мы исследовали положительно определенную эрмитову 4-метрику (7). Нас интересовал вопрос нахождения условий на коэффициенты этой метрики $a, b, u, v$, при которых ее тензор Римана является дуальным (автодуальным или антиавтодуальным). Всякая метрика с дуальным тензором Римана имеет нулевой тензор Риччи $R_{i j}=0$, то есть удовлетворяет вакуумным уравнениям Эйнштейна, поэтому данный вопрос имеет не только геометрический интерес. Решить задачу нахождения условий дуальности тензора Римана напрямую не представляется возможным, потому что компоненты тензора Римана, выражающиеся через частные производные 2-го порядка от функций $a, b, u, v$, оказываются очень громоздкими. Поэтому мы воспользовались методом, который заменяет очень сложные условия дуальности на значительно более простую систему дифференциальных уравнений в частных производных 1-го порядка, а именно на требование дуальности связности. Уравнения дуальности тензора Римана являются следствием этой системы, но в общем случае не равносильны ей. Поэтому, хотя нами и найдено общее решение уравнений автодуальности связности, мы не можем утверждать, что получили все эрмитовы метрики с автодуальным тензором Римана. Тем не менее, решая проблему дуальности тензора Римана для эрмитовой метрики, мы получили 5 новых решений вакуумных уравнений Эйнштейна в квадратурах или в явном виде. Все наши антиавтодуальные решения дают гиперкэлеровы метрики, в то время как ав- 
тодуальные решения приводят к гиперкэлеровой метрике только в плоском случае, когда тензор Римана равен нулю.

Конкурирующие интересы. Конкурирующих интересов не имеем.

Авторский вклад и ответственность. Все авторы принимали участие в разработке концепции статьи; все авторы сделали эквивалентный вклад в подготовку публикации. Авторы несут полную ответственность за предоставление окончательной рукописи в печать. Окончательная версия рукописи была одобрена всеми авторами.

Финансирование. Исследование выполнялось без финансирования.

Благодарность. Авторы благодарны рецензентам за тщательное прочтение статьи, ценные предложения и комментарии.

\section{Библиографический список}

1. Besse A. L. Einstein Manifolds. Berlin: Springer-Verlag, 1987. xii +512 pp. https://doi. org/10.1007/978-3-540-74311-8.

2. Atiyah M. F., Hitchin N. The Geometry and Dynamics of Magnetic Monopoles / Porter Lectures / Princeton Legacy Library. vol. 11. Princeton, New Jersey: Princeton University Press, 1988. vii+134 pp. https://doi.org/10.1515/9781400859306.

3. Петров А. 3. Новые методъ в общей теории относительности. М.: Наука, 1966. 496 с.

4. Eguchi T., Gilkey P. B., Hanson A. J. Gravitation, gauge theories and differential geometry// Phys. Reports, 1980. vol.66, no.6. pp. 213-393. https://doi.org/10.1016/ 0370-1573(80)90130-1.

5. Eguchi T., Hanson A. J. Asymptotically flat self-dual solutions to euclidean gravity // Phys. Lett. B, 1978. vol. 74, no. 3. pp. 249-251. https://doi.org/10.1016/0370-2693(78) 90566-X.

6. Gibbons G. W., Hawking S. W. Gravitational multi-instantons // Phys. Lett. B, 1978. vol. 78, no. 4. pp. 430-432. https://doi.org/10.1016/0370-2693(78)90478-1.

7. Koshti S., Dadhich N. The general self-dual solution of the Einstein equations, 1994. 14 pp., arXiv: gr-qc/9409046.

8. Кривоносов Л. Н., Лукьянов В. А. Специфика классификации Петрова (анти)автодуальных метрик нулевой сигнатуры // Изв. вузов. Матем., 2020. № 9. С. 56-67. https://doi .org/10.26907/0021-3446-2020-9-56-67.

9. Weil A. Introduction à L'Étude des Variétés Kähleriennes. Paris: Hermann, 1958. 175 pp. (In French)

10. Hitchin N. Compact four-dimensional Einstein manifolds // J. Differential Geom., 1974. vol. 9, no. 3. pp. 435-441. https://doi.org/10.4310/jdg/1214432419.

11. Hitchin N. J. Kählerian twistor spaces // Proc. London Math. Soc., 1981. vol. 43, no. 1. pp. 133-150. https://doi.org/10.1112/plms/s3-43.1.133.

12. Chen B.-Y. Some topological obstructions to Bochner-Kaehler metrics and their applications// J. Differential Geom., 1978. vol.13, no.4. pp. 547-558. https://doi.org/ $10.4310 / \mathrm{jdg} / 1214434707$.

13. Derdziński A. Self-dual Kähler manifolds and Einstein manifolds of dimension four // Compos. Math., 1983. vol. 49, no. 3. pp. 405-433 http://eudml.org/doc/89617.

14. Itoh M. Self-duality of Kähler surfaces // Compos. Math., 1984. vol.51, no. 2. pp. 265-273 http://eudml.org/doc/89645.

15. Jelonek W. Compact Kähler surfaces with harmonic anti-self-dual Weyl tensor // Diff. Geom. Appl., 2002. vol. 16, no. 3. pp. 267-276. https://doi.org/10.1016/S0926-2245(02) 00076-1.

16. Арсеньева О. Е. Автодуальная геометрия обобщенных келеровых многообразий // Maтем. сб., 1993. Т. 184, № 8. С. 137-148. 


\title{
MSC: 53B30, 58A14, 53C18
}

\section{Hermitian metrics with (anti-)self-dual Riemann tensor}

\author{
L. N. Krivonosov, V.A. Luk'yanov \\ Nizhny Novgorod State Technical University, \\ 24, Minina st., Nizhnii Novgorod, 603600, Russian Federation.
}

\begin{abstract}
Equations of (anti-)self-duality for the components of the Levi-Civita connection of the Hermitian positive definite metric (not for the Riemann tensor) are compiled. With this well-known method, a simpler system of partial differential equations is obtained, which implies the (anti-)self-duality of the Riemann tensor. This system is of the 1st order, while the (anti-)selfduality conditions of the Riemann tensor are expressed by equations of the 2nd order. However, this method can obtain only particular solutions of the (anti-)self-duality equations of the Riemann tensor. The constructed equations turned out to be significantly different in the self-dual and anti-self-dual cases. In the case of self-duality, the equations are divided into three classes, for each of which a general solution is found. In the anti-self-dual case, we did not find the general solution, but gave two series of particular solutions. The connection between our solutions and Kähler metrics is shown. In the case of the (anti-)self-duality of the Levi-Civita connection for the Hermitian metric, a general form of parallel almost complex metric-preserving structures is obtained. These structures are all torsion free. For an arbitrary positive definite 4-metric, a general form of almost complex structures preserving this metric is found.
\end{abstract}

Keywords: (anti-)self-duality, Hodge operator, Einstein vacuum equations of gravitation, Riemann tensor, Hermitian, Kähler, hyper-Kähler metric.

Received: $16^{\text {th }}$ June, $2021 /$ Revised: $18^{\text {th }}$ September, $2021 /$ Accepted: $12^{\text {th }}$ October, $2021 /$ First online: $16^{\text {th }}$ November, 2021

\section{Research Article}

(C) Authors, 2021

(C) Samara State Technical University, 2021 (Compilation, Design, and Layout)

ㅇ (ㅇ) The content is published under the terms of the Creative Commons Attribution 4.0 International License (http://creativecommons.org/licenses/by/4.0/)

Please cite this paper in press as:

Krivonosov L. N., Luk' yan ov V. A. Hermitian metrics with (anti-)self-dual Riemann tensor, Vestn. Samar. Gos. Tekhn. Univ., Ser. Fiz.-Mat. Nauki [J. Samara State Tech. Univ., Ser. Phys. Math. Sci.], 2021, vol. 25, no. 4, pp. 616-633. https://doi.org/10.14498/vsgtu1867 (In Russian).

\section{Authors' Details:}

Leonid N. Krivonosov (10) https://orcid.org/0000-0002-3533-9595

Cand. Phys. \& Math. Sci.; Associate Professor; Dept. of Applied Mathematics; e-mail: 1.n.krivonosov@gmail.com

Vyacheslav A. Luk'yanov (10) https://orcid.org/0000-0002-7294-0232

Cand. Phys. \& Math. Sci.; Associate Professor; Dept. of Applied Mathematics; e-mail: oxyzt@ya.ru 
Competing interests. The authors declare no conflicts of interests.

Authors' contributions and responsibilities. Each author has participated in the article concept development; the authors contributed equally to this article. The authors are absolutely responsible for submit the final manuscript to print. Each author has approved the final version of manuscript.

Funding. This research received no specific grant from any funding agency in the public, commercial, or not-for-profit sectors.

Acknowledgments. The authors are grateful to referees for their careful reading, suggestions and valuable comments.

\section{References}

1. Besse A. L. Einstein Manifolds. Berlin, Springer-Verlag, 1987, xii+512 pp. https://doi. org/10.1007/978-3-540-74311-8.

2. Atiyah M. F., Hitchin N. The Geometry and Dynamics of Magnetic Monopoles, Porter Lectures / Princeton Legacy Library, vol. 11. Princeton, New Jersey, Princeton University Press, 1988, vii+134 pp. https://doi.org/10.1515/9781400859306.

3. Petrov A. Z. Novye metody v obshchei teorii otnositel'nosti [New Methods in General Relativity]. Moscow, Nauka, 1966, 496 pp. (In Russian)

4. Eguchi T., Gilkey P. B., Hanson A. J. Gravitation, gauge theories and differential geometry, Phys. Reports, 1980, vol.66, no. 6, pp. 213-393. https://doi.org/10.1016/0370-1573(80) 90130-1.

5. Eguchi T., Hanson A. J. Asymptotically flat self-dual solutions to euclidean gravity, Phys. Lett. B, 1978, vol.74, no. 3, pp. 249-251. https://doi.org/10.1016/0370-2693(78) 90566-X.

6. Gibbons G. W., Hawking S. W. Gravitational multi-instantons, Phys. Lett. B, 1978, vol. 78, no. 4, pp. 430-432. https://doi.org/10.1016/0370-2693(78)90478-1.

7. Koshti S., Dadhich N. The general self-dual solution of the Einstein equations, 1994, 14 pp., arXiv: gr-qc/9409046.

8. Krivonosov L. N., Luk'yanov V. A. Specificity of Petrov classification of (anti-)self-dual zero signature metrics, Russian Math. (Iz. VUZ), 2020, vol.64, no.9, pp. 50-60. https://doi. org/10.3103/S1066369X20090054.

9. Weil A. Introduction à L'Étude des Variétés Kähleriennes. Paris, Hermann, 1958, 175 pp. (In French)

10. Hitchin N. Compact four-dimensional Einstein manifolds, J. Differential Geom., 1974, vol. 9, no. 3, pp. 435-441. https://doi.org/10.4310/jdg/1214432419.

11. Hitchin N. J. Kählerian twistor spaces, Proc. London Math. Soc., 1981, vol. 43, no. 1, pp. 133150. https://doi.org/10.1112/plms/s3-43.1.133.

12. Chen B.-Y. Some topological obstructions to Bochner-Kaehler metrics and their applications, J. Differential Geom., 1978, vol.13, no.4, pp. 547-558. https://doi.org/ $10.4310 / \mathrm{jdg} / 1214434707$.

13. Derdziński A. Self-dual Kähler manifolds and Einstein manifolds of dimension four, Compos. Math., 1983, vol.49, no. 3, pp. 405-433 http://eudml.org/doc/89617.

14. Itoh M. Self-duality of Kähler surfaces, Compos. Math., 1984, vol.51, no. 2, pp. 265-273 http://eudml.org/doc/89645.

15. Jelonek W. Compact Kähler surfaces with harmonic anti-self-dual Weyl tensor, Diff. Geom. Appl., 2002, vol.16, no.3, pp. 267-276. https://doi.org/10.1016/S0926-2245(02) 00076-1.

16. Arsen'eva O. E. Selfdual geometry of generalized Kählerian manifolds, Russian Acad. Sci. Sb. Math., 1994, vol.79, no.2, pp. 447-457. https://doi.org/10.1070/ SM1994v079n02ABEH003509. 